\title{
Observation of the curative effect of intraocular lens in patients with cataract and establishment of the intraocular lens database
}

\author{
Suhui $\mathrm{Xu}^{1}$, Yuen Liang ${ }^{2}$, Haiyan $\mathrm{Cai}^{1}$, Yue Ying ${ }^{3}$, Meijuan $\mathrm{Lan}^{4}$ \\ ${ }^{1}$ Ophthalmology Operating Room, Second Affiliated Hospital of Zhejiang University College of Medicine, Hangzhou, China; ${ }^{2}$ Department of \\ Modern Information Technology College, Zhejiang Mechanical and Electrical Vocational College, Hangzhou, China; ${ }^{3}$ Department of Clinical \\ Engineering, Second Affiliated Hospital of Zhejiang University School of Medicine, Hangzhou, China; ${ }^{4}$ Department of Nursing, Second Affiliated \\ Hospital of Zhejiang University School of Medicine, Hangzhou, China \\ Contributions: (I) Conception and design: S Xu, Y Liang; (II) Administrative support: M Lan; (III) Provision of study materials or patients: Y Ying; (IV) \\ Collection and assembly of data: All authors; (V) Data analysis and interpretation: S Xu, M Lan; (VI) Manuscript writing: All authors; (VII) Final \\ approval of manuscript: All authors. \\ Correspondence to: Meijuan Lan. Department of Nursing, Second Affiliated Hospital of Zhejiang University School of Medicine, Hangzhou 310007, \\ China. Email: lanmj@zju.edu.cn.
}

\begin{abstract}
Backgroundk Intraocular lenses are widely used in the treatment of cataract patients, but currently clinical, procedural and product information is incomplete in many hospitals and there is an urgent need for a database to improve surgical safety, and outcomes.

Methods: Data on the brand and specifications of the intraocular lens, and the postoperative outcome of cataract patients after intraocular lens implantation from January 2017 to June 2018 were collected to create an intraocular lens database as an online public platform.

Results: A total of 96 cataract patients were divided into groups A and B based on preoperative corrected visual acuity. At 3 months after surgery the visual acuity of patients in group A recovered from $1.51 \pm 0.19$ to $0.76 \pm 0.18$, and the in group B it recovered from $1.02 \pm 0.27$ to $0.49 \pm 0.13$. Overall, the postoperative visual acuity of all patients improved significantly $(\mathrm{P}<0.001)$. We also used the NEI-VFQ-25 visual function questionnaire to evaluate the visual function of the patients before and after surgery. At 3 months after surgery, the mean score of patients in group A increased from $21.89 \pm 14.21$ to $59.97 \pm 18.29$ and in group B it increased from $38.26 \pm 17.57$ to $70.28 \pm 12.37$. Overall, the patients' postoperative scores increased significantly $(\mathrm{P}<0.001)$.
\end{abstract}

Conclusions: Cataract phacoemulsification and intraocular lens implantation can effectively and safely restore and improve the visual function of cataract patients. Information sharing through establishment of an intraocular lens information database will assist in further advances and consistency in the treatment of cataract patients.

Keywords: Cataract; intraocular lens; visual function; NEI-VFQ-25

Submitted Feb 24, 2021. Accepted for publication Apr 17, 2021.

doi: 10.21037/apm-21-632

View this article at: http://dx.doi.org/10.21037/apm-21-632

\section{Introduction}

Cataract is a common ophthalmologic disease that causes blurred vision due to lens protein degeneration and turbidity caused by lens metabolic disorder. The main surgical methods of treatment are phacoemulsification and intraocular lens implantation $(1,2)$. Intraocular lenses are widely used in the treatment of senile cataract, concurrent cataract, aphakia, lens dislocation, and high myopia (3). However, currently, hospitals in China have incomplete records of the brand, specifications, performance, materials, and other information of the intraocular lenses used in patients 
undergoing intraocular lens implantation. As a result, when the other eye requires cataract surgery or the patient goes to another hospital for the procedure, the information is not linked, which can affect the surgical outcome. Therefore, it is urgent and important to establish a convenient and complete information system and public network platform, implement information interconnection to improve surgical safety, and help surgeons and cataract patients by providing relevant information. We present the following article in accordance with the STROBE reporting checklist (available at http:// dx.doi.org/10.21037/apm-21-632).

\section{Methods}

\section{General information}

A total of 96 cataract patients (96 eyes) who underwent phacoemulsification and intraocular lens implantation at Second Affiliated Hospital of Zhejiang University College of Medicine from January 2017 to June 2018 were selected, comprising 54 male patients and 42 female patients, with an average age of $(53.26 \pm 6.12)$ years old. The diagnosis of cataract was based on the lens opacity classification system II (LOCS II) (4) and the 2008 American Ophthalmology Clinical Guidelines. Exclusion criteria were: (I) eye diseases such as corneal disease, glaucoma, and retinal detachment; (II) history of internal and external ophthalmic surgery; (III) Intraocular lens implantation surgical complications; (IV) unwillingness to cooperate with the requirements of this study. All procedures performed in accordance with the Declaration of Helsinki (as revised in 2013). Informed consent was taken from all the patients. The study protocol was approved by the institutional ethics committee of the Second Affiliated Hospital of Zhejiang University College of Medicine (No.20160041).

\section{Preoperative examination}

Routine preoperative examinations of vision, intraocular pressure, fundus, ophthalmological A/B ultrasound, etc. were performed. The refractive power of the intraocular lens was calculated according to the SBK-K formula, and the corresponding intraocular lens was customized for of each patient.

\section{Surgical procedure}

Cataract phacoemulsification and intraocular lens implantation were performed under surface anesthesia. After routine disinfection, the eyelid opener was used to open the eyelid without fixing the superior rectus muscle. Next, a 1-mm incision was made in the limbus with a corneal puncture knife at about 3 o'clock, and the viscoelastic agent was injected into the anterior chamber to maintain the normal depth of the chamber. A $3.0-\mathrm{mm}$ or small $1.8-\mathrm{mm}$ tunnel incision was made in the transparent cornea at 10 11 o'clock, followed by continuous circular capsulorhexis performed with capsulorhexis forceps; balanced salt solution (BSS) was used for capsule-cortex-nuclear water separation. Use both hands to emulsify the cataract, a 1/A system was then used to infuse and aspirate the residual cortex while polishing the posterior capsule. After sodium hyaluronate was injected into the anterior chamber and capsular bag, the diameter of the optical part of the implanted intraocular lens was enlarged to 5.5 or $6 \mathrm{~mm}$. Lens forceps were used to implant the intraocular lens into the capsular bag as usual, the anterior chamber was flushed with BSS, and the residual sodium hyaluronate was extracted by suction. Finally, BSS was injected into the anterior chamber, until the corneal incision became watertight without suturing. Finally, amikacin and dexamethasone were injected subconjunctivally, and the eye was bandaged. On the first postoperative day, tobramycin dexamethasone eye drops were applied, and tobramycin dexamethasone ointment was applied every night. Oral hormones were taken for 3-4 days depending on the intraoperative reaction.

\section{Surgical efficacy}

\section{Vision correction}

The standard logarithmic visual acuity chart was used to calculate the logarithmic expression of the minimum angle of resolution (logMAR). Of the 96 patients, 38 with preoperative corrected visual acuity $(\log M A R) \geq 1.3$ were designated as group $\mathrm{A}$, and 58 with $\log \mathrm{MAR}$ between 0.5 and 1.3 were group $\mathrm{B}$. The pre- and 3 -month postoperative $\log$ MAR values of groups A and B groups were recorded.

\section{Visual function questionnaire}

The 25-item National Eye Institute Visual Function Questionnaire (NEI-VFQ-25) $(5,6)$ was used to subjectively evaluate the visual function of patients before and 3 months after surgery. The questionnaire comprises 1 item for general health status, 1 item for total visual condition, 2 items for eye pain, 3 items for close work, 3 items for longdistance work, 2 items for social functioning, 4 items for 
Table 1 Comparison of visual acuity of two groups of cataract patients before and after surgery (n, $\bar{x} \pm s)$

\begin{tabular}{lccccc}
\hline Group & $\mathrm{n}$ & Preoperative $(\log M A R)$ & 3 months after surgery (logMAR) & $t$ value & $P$ value \\
\hline A & 38 & $1.51 \pm 0.19$ & $0.76 \pm 0.18$ & 17.665 & $<0.001$ \\
B & 58 & $1.02 \pm 0.27$ & $0.49 \pm 0.13$ & 13.470 & $<0.001$ \\
\hline
\end{tabular}

Table 2 Comparison of NEI-VFQ-25 scores of two groups of cataract patients before and after surgery (n, $\bar{x} \pm \mathrm{s})$

\begin{tabular}{lccccc}
\hline Group & $\mathrm{n}$ & Preoperative & 3 months after surgery & $t$ value & P value \\
\hline A & 38 & $21.89 \pm 14.21$ & $59.97 \pm 18.29$ & -10.135 & $<0.001$ \\
B & 58 & $38.26 \pm 17.57$ & $70.28 \pm 12.37$ & -11.349 & $<0.001$ \\
\hline
\end{tabular}

mental health status, 2 items for social role restriction, 3 items for independence, 1 item for color vision and 1 item for peripheral vision. The scoring method was $0-4$ for each item, and a full score of 100 . The higher the total score, the better the quality of life related to vision.

\section{Statistical analysis}

All data were processed with SPSS 22.0 statistical software. The count data are described by examples (n) and the average \pm standard deviation $(\bar{x} \pm \mathrm{s}) ; t$-test ( $t$ value) was used for statistical analysis between groups. $\mathrm{P}<0.05$ indicates a significant statistical difference, and $\mathrm{P}<0.001$ indicates a very significant statistical difference.

\section{Results}

\section{Visual acuity}

According to their logMAR values, the difference in corrected visual acuity in groups A and B before and after surgery was statistically significant (Table $1, \mathrm{P}<0.001$ ).

\section{NEI-VFQ-25 scores}

The NEI-VFQ-25 scores for groups A and B are presented in Table 2. The difference in the scores between before and after surgery was statistically significant $(\mathrm{P}<0.001)$.

\section{Establishment of the database}

Prior to the establishment of a dedicated database for intraocular lens implantation, information about the lens being used was provided by surgeon before the operation, in addition to the relevant implantable material information system in the hospital. After the operation, archived medical records and outpatient medical records were attached to the label provided by the manufacturer of the lens, which included the brand and other limited information, depending on the size of the label.

For each of the 96 cataract patients, we recorded the preoperative examination results, age, refractive power of the intraocular lens was determined and the most suitable type of intraocular lens for the patient. The intraocular lenses used in this study were classified according to the surface attachment, brand, type, and the shape of the loop of the intraocular lens. Details are as follows.

(I) Surface attachment: 35 cases of polymethyl methacrylate and 61 cases of silicone gel.

(II) Brand: 15 American ALCON, 23 American Bausch \& Lomb, 6 British RAYNER, 4 Japan Itabashi Canon, 5 Japan HOYA, 20 Henan Universe, 23 Zhuhai Aige.

(III) Lens type: 20 single-focus intraocular lenses; 14 diffractive multifocal intraocular lenses (AcrysofReSTOR or Tecnis diffractive multifocal); 10 astigmatic single-focus intraocular lenses (AcrysofToric, TecnisToric); 4 adjustable intraocular lenses; 40 trifocal intraocular lens; 8 new electrodeless intraocular lens (AMO Symfone).

(IV) Shape of the loop: $44 \mathrm{C}$ type, $43 \mathrm{~J}$ type, 9 CJ type.

(V) The database included the personal information of patients who gave informed consent for their personal information to be uploaded, including their name, ID number, surgical procedure (including which eye), and the name of the surgeon, and related information about the intraocular lens such as brand, specifications, lens power, manufacturer, etc. 


\section{Discussion}

Cataract is a common ophthalmology disease that causes lens protein degeneration and turbidity due to lens metabolism disorder, which leads to blurred vision. It mostly occurs in people over 40 years of age $(7,8)$. Cataract treatment includes drug treatment, phacoemulsification, extracapsular cataract extraction, and other methods (9). Of these, phacoemulsification and intraocular lens implantation are widely used clinically because of the small surgical incision, minimal tissue trauma, fast postoperative recovery of vision, and relatively few surgical complications $(2,10)$. Among the 96 cataract patients of this study, visual acuity of all patients improved significantly after surgery $(\mathrm{P}<0.001)$, and the NEI-VFQ-25 visual function survey showed a statistically significant difference $(\mathrm{P}<0.001)$ between the pre-and postoperative scores. All these results demonstrated that phacoemulsification and intraocular lens implantation can restore vision and improve the visual function of cataract patients.

The ophthalmic surgeon will choose the most suitable intraocular lens suitable for the patient based on their individual needs (11-16), but over time patients will forget the specific brand, specifications, performance, materials, and power of the implanted lens. If the patient's other eye also needs cataract surgery, the surgeon needs the information about the implanted lens to select the appropriate implant for the current intraocular lens specification, model, performance, parameters, etc. For patients with different degrees of cataract in both eyes, traumatic cataract in one eye, economic constraints, etc., patients who have a long interval between operations on both eyes, or who underwent cataract surgery on the other eye at another hospital, it is often difficult to obtain information about the intraocular lens implanted in the other eye, which will affect the postoperative visual effect. In serious cases, due to the implantation of intraocular lenses of different materials and properties in both eyes, the difference in binocular vision can lead to dizziness, nausea, and glare, which will affect the quality of life. In addition, when intraocular lens patients need intraocular lens replacement due to postoperative complications, ocular trauma, or poor postoperative visual effects, or an intraocular lens was added to an intraocular lens due to vision changes (the previously implanted intraocular lens was re-implanted due to decreased vision, namely double lens implantation), or because of certain diseases such as retinopathy, or intraocular silicone oil filling was required, detailed information about the lens and the procedure is required. Therefore, the establishment of a comprehensive intraocular lens information database for patients and professional institutions to consult will meet this need. Our data for the 96 study patients formed the basis for a public database of intraocular lens information. All the information about the user's cataract surgery and the intraocular lens used can be accessed by logging in to an account registered with the user's identity information, which is very convenient for both doctors and patients, will improve surgical safety and medical treatment, and maximize the benefits of cataract surgery for patients. In addition, surgical and intraocular lens information is stored in the hospital database, which has extremely high security and will also provide patients with continuity of service.

As the number of cataract procedures increases, the information in the database will gradually increase, further enriching the information source, and providing a safe information depository and continuation of service for current and subsequent cataract patients.

\section{Acknowledgments}

Funding: The study was supported by Zhejiang Province Medicine and Health Science and Technology Program Project Applied Research (2017187993).

\section{Footnote}

Reporting Checklist: The authors have completed the STROBE reporting checklist. Available at http://dx.doi. org/10.21037/apm-21-632

Data Sharing Statement: Available at http://dx.doi. org/10.21037/apm-21-632

Conflicts of Interest: All authors have completed the ICMJE uniform disclosure form (available at http://dx.doi. org/10.21037/apm-21-632). The authors have no conflicts of interest to declare.

Etbical Statement: The authors are accountable for all aspects of the work in ensuring that questions related to the accuracy or integrity of any part of the work are appropriately investigated and resolved. All procedures performed in this study were in accordance with the Declaration of Helsinki (as revised in 2013). Informed consent was taken from all the patients. The study protocol 
was approved by the ethics committee of the Second Affiliated Hospital of Zhejiang University College of Medicine (No. 20160041).

Open Access Statement: This is an Open Access article distributed in accordance with the Creative Commons Attribution-NonCommercial-NoDerivs 4.0 International License (CC BY-NC-ND 4.0), which permits the noncommercial replication and distribution of the article with the strict proviso that no changes or edits are made and the original work is properly cited (including links to both the formal publication through the relevant DOI and the license). See: https://creativecommons.org/licenses/by-nc-nd/4.0/.

\section{References}

1. Kałuzny J, Biardzka B, Wiliński M. Cataract in the only eye. 2. Surgical treatment and its results. Klin Oczna 1982;84:221-2.

2. Ao MX, Wang W, Li XM, et al. Changes of dynamic visual acuity after phacoemulsification combined with intraocular lens implantation. Zhonghua Yan Ke Za Zhi 2013;49:405-9.

3. Zhao Y, Yang K, Li J, et al. Comparison of hydrophobic and hydrophilic intraocular lens in preventing posterior capsule opacification after cataract surgery: An updated meta-analysis. Medicine (Baltimore) 2017;96:e8301.

4. Klein BE, Klein R, Linton KL, et al. Assessment of cataracts from photographs in the Beaver Dam Eye Study. Ophthalmology 1990;97:1428-33.

5. Mangione CM, Lee PP, Gutierrez PR, et al. Development of the 25-item National Eye Institute Visual Function Questionnaire. Arch Ophthalmol 2001;119:1050-8.

6. Wang CW, Chan CL, Jin HY. Psychometric properties of the Chinese version of the 25-item National Eye Institute Visual Function Questionnaire. Optom Vis Sci 2008;85:1091-9.

7. Zhu Y, Chen X, Chen P, et al. The occurrence rate of acute-onset postoperative endophthalmitis after cataract surgery in Chinese small- and medium-scale departments of ophthalmology. Sci Rep 2017;7:40776.

Cite this article as: $\mathrm{Xu} \mathrm{S,} \mathrm{Liang} \mathrm{Y,} \mathrm{Cai} \mathrm{H,} \mathrm{Ying} \mathrm{Y,} \mathrm{Lan} \mathrm{M.}$ Observation of the curative effect of intraocular lens in patients with cataract and establishment of the intraocular lens database. Ann Palliat Med 2021;10(4):4716-4720. doi: 10.21037/apm-21-632
8. Artini W, Artini W, Gondhowiarjo TD, et al. Impacts of Impaired Vision and Eye Diseases on Vision-Related Quality of Life in Indonesia. Makara Journal of Health Research 2017;21:104-10.

9. Chan WH, Biswas S, Ashworth JL, et al. Congenital and infantile cataract: aetiology and management. Eur J Pediatr 2012;171:625-30.

10. Chelerkar V, Parekh P, Kalyani VKS, et al. Comparative Clinical Study of Medically Controlled Nonsevere Chronic Primary Angle-closure Glaucoma with Coexisting Cataract Surgically Managed by Phacoemulsification as against Combined Phacotrabeculectomy. Middle East Afr J Ophthalmol 2018;25:119-25.

11. Barisić A, Gabrić N, Dekaris I, et al. Comparison of different presbyopia treatments: refractive lens exchange with multifocal intraocular lens implantation versus LASIK monovision. Coll Antropol 2010;34 Suppl 2:95-8.

12. Vilar C, Hida W'T, de Medeiros AL, et al. Comparison between bilateral implantation of a trifocal intraocular lens and blended implantation of two bifocal intraocular lenses. Clin Ophthalmol 2017;11:1393-7.

13. Monaco G, Gari M, Di Censo F, et al. Visual performance after bilateral implantation of 2 new presbyopia-correcting intraocular lenses: Trifocal versus extended range of vision. J Cataract Refract Surg 2017;43:737-47.

14. Orczykowska M, Owidzkaz M, Synder A, et al. Comparative analysis of early distance visual acuity in patients after coaxial phacoemulsification through the micro-incision $(1.8 \mathrm{~mm})$ and after standard phacoemulsification through the small incision $(2.75 \mathrm{~mm})$. Klin Oczna 2014;116:7-10.

15. Yang JJ, Liu QP, Li JM, et al. Comparison of visual outcomes with implantation of trifocal versus bifocal intraocular lens after phacoemulsification: a Meta-analysis. Int J Ophthalmol 2018;11:484-92.

16. Jain S, Rajshekar K, Aggarwal A, et al. Effects of cataract surgery and intra-ocular lens implantation on visual function and quality of life in age-related cataract patients: a systematic review protocol. Syst Rev 2019;8:204.

(English Language Editor: K. Brown) 